\section{A) Check for updates}

Cite this: Food Funct., 2019, 10, 7952

\title{
Characterisation of peroxidation products arising from culinary oils exposed to continuous and discontinuous thermal degradation processes $\uparrow$
}

\author{
Adam Le Gresley, (D) *a Gilbert Ampem, ${ }^{a}$ Martin Grootveld, ${ }^{\mathrm{b}}$ Benita C. Percival ${ }^{\mathrm{b}}$ and \\ Declan P. Naughton ${ }^{a}$
}

High-resolution NMR analysis has been used, for the first time, to identify, putatively, two new secondary aldehydic lipid oxidation products in culinary oils. The impact of heating and cooling times on the thermal stability, fatty acid composition and lipid oxidation product (LOP) concentrations have been analysed for continuous and discontinuous heating periods $\left(180^{\circ} \mathrm{C}\right)$. The susceptibility of the selected oils to thermal oxidation for the different heating episodes has been evaluated via the detection and determination of LOPs, particularly cytotoxic and genotoxic aldehydes. The identities and quantities of these LOPs evolved throughout a 2.0 hour period. Results acquired indicated that sunflower oil was more resistant to discontinuous oxidation than rapeseed and olive oils, however overall discontinuous heating resulted in more LOPs.

Received 5th September 2019 Accepted 18th November 2019 DOI: $10.1039 /$ c9fo02065a rsc.li/food-function the evolution of LOPs during such thermal stressing periods conducted according to standard frying practices, specifically:

- The relationship between unsaturation status and susceptibility to thermal oxidation;

- The relationship between oil type and the identity of the LOPs evolved during thermal oxidation processes;

- The quantity of LOPs generated according to the type of thermal oxidation process that culinary oils frequently undergo.

Through the identification and quantification of LOPs produced in different types of thermo-oxidation approaches, it may be possible to identify the best oils and/or methods of heating in order to reduce the concentrations and classes of LOPs generated. This has the potential to aid in the prevention of emerging public health issues in humans associated with the dietary ingestion and/or inhalation of LOPs. ${ }^{5}$

\subsection{Chemical changes to fatty acid (FA) chain composition}

During thermal oxidation, both major and minor acyl groups of culinary fats and oils are degraded at variable rates to produce new compounds. ${ }^{6}$

It has already been established that the intensities of signals generated in the ${ }^{1} \mathrm{H}$ NMR spectra (Fig. 1) is proportional to the product of the number of protons that give rise to them and the concentration(s) of the assigned molecule (s) featured, and quantification can be performed via comparisons to the intensity of a validated internal reference standard. ${ }^{6,7}$ The calculations involved in this process for the quantification of the FAs indicated in Table 1 are shown in ESI (Summary S1). $\dagger$ In view of established literature, ${ }^{1} \mathrm{H}$ NMR analysis was selected as a non-destructive, quantitative technique,

\footnotetext{
${ }^{a}$ Department of Chemistry and Pharmaceutical Sciences, SEC Faculty, Kingston University, Kingston-upon-Thames, Surrey, KT1 2EE, UK.

E-mail: a.legresley@kingston.ac.uk; Tel: +44 (0)2084177432

${ }^{b}$ Health and Life Sciences, De Montfort University, Leicester, LE1 9BH, UK

$\dagger$ Electronic supplementary information (ESI) available. See DOI: 10.1039/ c9fo02065a
} 


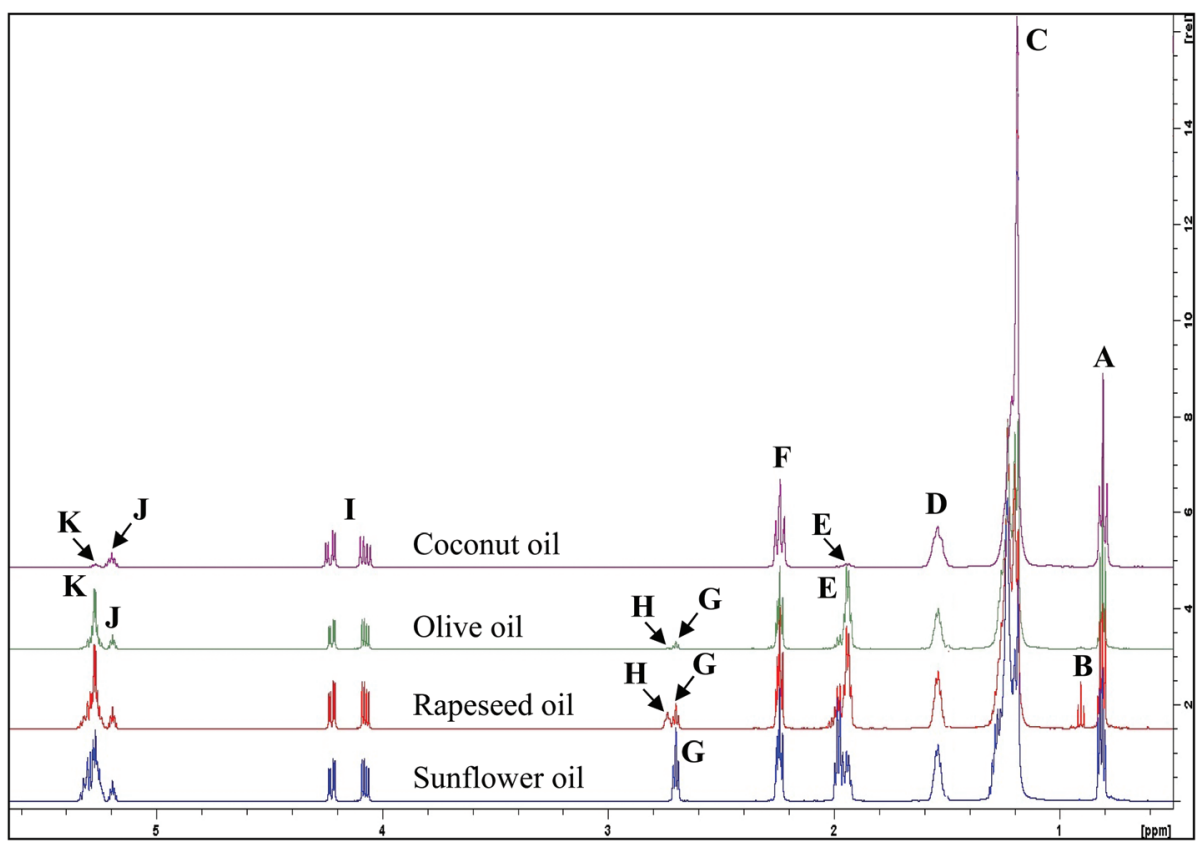

Fig. $1{ }^{1} \mathrm{H}$ NMR spectra of major acylglycerol groups present in the $0.0-5.4 \mathrm{ppm}$ regions of unheated culinary oils. The letter assignments of resonances correspond to those provided in Table 1.

Table 1 Assignment of bulk ${ }^{1} \mathrm{H}$ NMR signals of acylglycerol groups present in the ${ }^{1} \mathrm{H}$ NMR profiles of unheated culinary oils, including chemical shift values, multiplicities and their associated functional groups

\begin{tabular}{|c|c|c|c|c|}
\hline \multirow[b]{2}{*}{ Signal } & \multirow[b]{2}{*}{ Chemical shift (ppm) } & \multirow[b]{2}{*}{ Multiplicity } & \multicolumn{2}{|l|}{ Functional group } \\
\hline & & & Condensed function & Classification \\
\hline A & $0.743-0.872$ & $\mathrm{t}$ & $-\mathrm{CH}_{3}$ & Saturated, oleic and linoleic acyl groups \\
\hline $\mathrm{B}$ & $0.872-0.936$ & $\mathrm{t}$ & $-\mathrm{CH}_{3}$ & Unsaturated $\omega-3$ acyl groups \\
\hline $\mathrm{C}$ & $1.116-1.347$ & $\mathrm{t}$ & $-\left(\mathrm{CH}_{2}\right)_{n}^{-}$ & Acyl groups \\
\hline $\mathrm{D}$ & $1.462-1.625$ & $\mathrm{~m}$ & $-\mathrm{OCO}-\mathrm{CH}_{2}-\mathrm{CH}_{2}^{-}$ & Acyl groups except for DHA, EPA and ARA acyl groups \\
\hline $\mathrm{E}$ & $1.859-2.056$ & $\mathrm{~m}$ & $-\mathrm{CH}_{2}-\mathrm{CH}=\mathrm{CH}-$ & $\begin{array}{l}\text { Acyl groups except for }-\mathrm{CH}_{2}-\text { of DHA acyl group in } \\
\beta \text {-position relative to the carbonyl function }\end{array}$ \\
\hline $\mathrm{F}$ & $2.172-2.304$ & $\mathrm{dt}$ & $-\mathrm{OCO}-\mathrm{CH}_{2}^{-}$ & Acyl groups except for DHA acyl groups \\
\hline G & $2.653-2.725$ & $\mathrm{t}$ & $=\mathrm{HC}-\mathrm{CH}_{2}-\mathrm{CH}=$ & Diunsaturated $\omega-6$ acyl groups \\
\hline $\mathrm{H}$ & $2.725-2.782$ & $\mathrm{t}$ & $=\mathrm{HC}-\mathrm{CH}_{2}-\mathrm{CH}=$ & Triunsaturated $\omega-3$ acyl groups \\
\hline I & $4.014-4.282$ & $\mathrm{dd}, \mathrm{dd}$ & $-\mathrm{CH}_{2} \mathrm{OCOR}$ & Glyceryl backbone groups \\
\hline $\mathrm{J}$ & $5.157-5.229$ & $\mathrm{~m}$ & $>\mathrm{CHOCOR}$ & Glyceryl backbone groups \\
\hline $\mathrm{K}$ & $5.229-5.368$ & $\mathrm{~m}$ & $-\mathrm{CH}=\mathrm{CH}-$ & Acyl chain olefinic functions \\
\hline
\end{tabular}

Abbreviations: d, doublet; t, triplet; m, multiplet; dd, double doublet; dt, double triplet; $\omega-3$, omega-3 acyl groups; $\omega-6$, omega-6 acyl groups; DHA, docosahexaenoyl acyl groups; EPA, eicosapentaenoyl acyl groups; ARA, arachidonoyl acyl groups. Letters assigned to signals correspond to those given in Fig. 1.

which is capable of identifying and quantifying components without the requirement for their prior separation.

\subsection{Oxidation products of FAs}

As a consequence of the thermal degradation of unsaturated acyl groups in culinary oil samples, many LOPs of varying chemical reactivity have been shown to evolve, ${ }^{8}$ and all types of LOPs found in this study are listed in Tables 2 and 3, with their respective spectra shown in Fig. 2, 3, 4, 5 and 6.

The thermal degradation of unsaturated acyl groups in culinary oil yields the evolution of primary LOPs. Primary LOPs are unstable, short-lived intermediates whose detection is often difficult to recount. ${ }^{9}$ Their degradation further leads to the formation of secondary LOPs, which are designated stable species and as a result, do pose a greater health risk to living cells and organs. Primary LOPs detected in this study are listed in Table 2 and their respective spectra shown on Fig. 2 and 3, as well as in Fig. S1 and S2 (ESI†).

Throughout the $300 \mathrm{~min}$ heating duration, conjugated hydroperoxydienes and hydroxymonoenes, and olefinic resonances of $\alpha, \beta$-UA, were observed to sequentially evolve with increasing heating time as shown in Fig. 2 for sunflower oil. 
Table 2 Assignment of ${ }^{1} \mathrm{H}$ NMR signals of conjugated hydroperoxydienes (CHPDs) and hydroxymonoenes (primary LOPs), and olefinic resonances of $\alpha, \beta$-unsaturated aldehydes present in the ${ }^{1} \mathrm{H}$ NMR profiles of sunflower oil thermally stressed continuously throughout a 300 min period. Chemical shift values, multiplicities and associated functional groups are shown

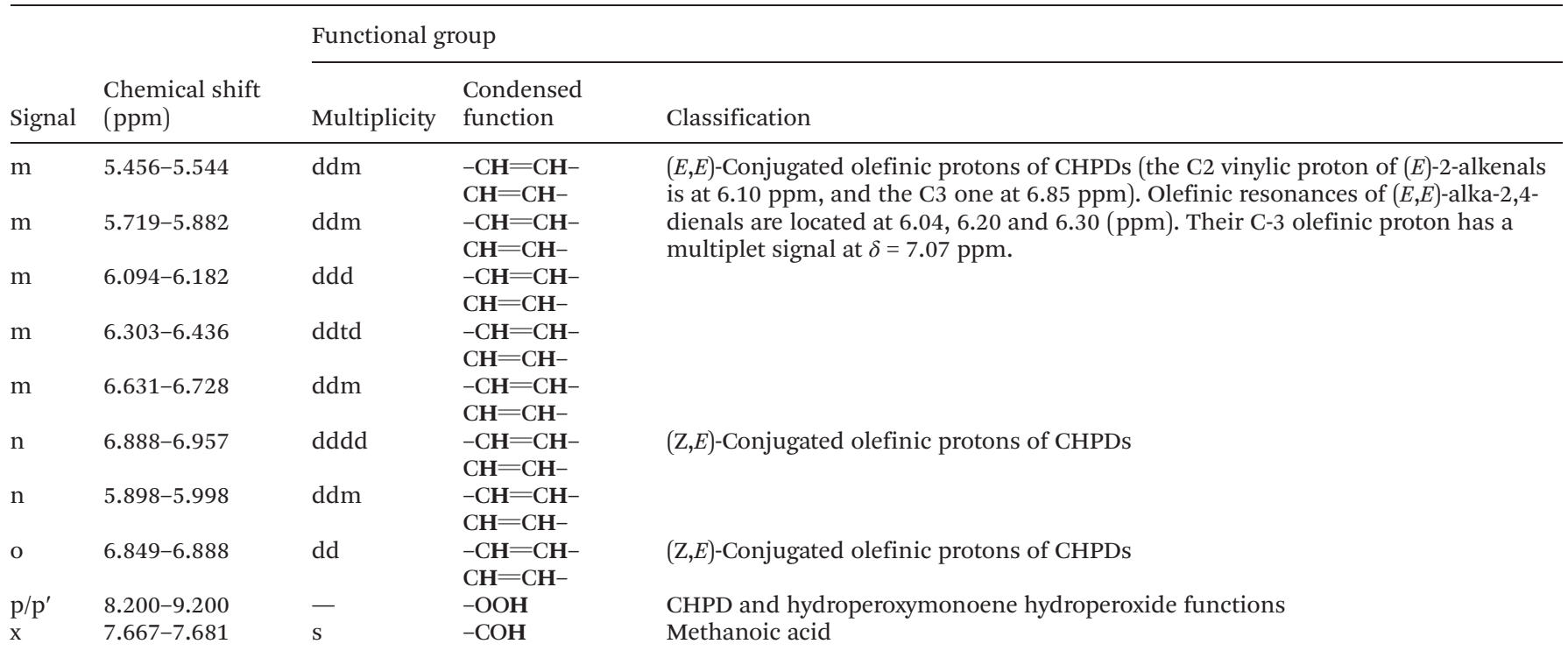

Abbreviations: s, singlet; d, doublet; t, triplet; m, multiplet; dd, double doublet; dt, double triplet. Letters assigned to signals correspond to those in Fig. 2 and 3.

Table 3 Assignment of ${ }^{1} \mathrm{H}$ NMR signals of epoxides (secondary LOPs) and primary alcohols present in the ${ }^{1} \mathrm{H}$ NMR profiles of sunflower oil thermally stressed continuously throughout a 300 min period. Chemical shifts, multiplicities and associated functional groups are shown

\begin{tabular}{lllll}
\hline & & & \multicolumn{2}{l}{ Functional group } \\
\cline { 5 - 5 } Signal & Chemical shift $(\mathrm{ppm})$ & Multiplicity & Condensed function & Classification \\
\hline $\mathrm{z}$ & $2.128-2.182$ & $\mathrm{~m}$ & - & Unidentified \\
$\mathrm{q}$ & $2.258-2.335$ & $\mathrm{~m}$ & $-\mathrm{CHOHC}-$ & $(E)-9,10$-Epoxystearate \\
$\mathrm{r}$ & $2.488-2.532$ & $\mathrm{~m}$ & $-\mathrm{CHOHC}-$ & $(Z)-9,10$-Epoxystearate \\
$\mathrm{s}$ & $2.532-2.568$ & $\mathrm{~m}$ & $-\mathrm{CHOHC}-\mathrm{CHOHC}-$ & $9,10-12,13$-Diepoxyoctadecanoate \\
$\mathrm{t}$ & $2.651-2.796$ & $\mathrm{~m}$ & $-\mathrm{CHOHC}-$ & 9,10 -Epoxy-octadecanoate; 9,10-Epoxy-12-octadecenoate \\
& & & & (leukotoxin); and 12,13-Epoxy-9-octadecenoate (isoleukotoxin) \\
$\mathrm{u}$ & $3.172-3.303$ & $\mathrm{~m}$ & $-\mathrm{CHOHC}_{2} \mathrm{CH}_{2}-\mathrm{CHOHC}-$ & $9,10-12,13-$ Diepoxyoctadecanoate \\
$\mathrm{v}$ & $3.548-3.595$ & $\mathrm{~m}$ & $\alpha-\mathrm{CH}_{2}$ & Primary alcohol LOPs
\end{tabular}

Abbreviations: m, multiplet. Letter assignments correspond to those provided in Fig. 4.

Amongst the culinary oils, the primary LOPs were predominantly low or undetectable in coconut oil (Fig. S1†). The concentrations of these primary LOPs were found to be similar for monounsaturated fatty acid (MUFA)-rich olive and rapeseed oils. Nonetheless, higher signals were observed in thermallystressed sunflower oil (Fig. S1†). The differences in the observable primary LOPs are ascribable to the differences in the UFA contents of the studied oils, i.e. sunflower oils has the highest content of particularly peroxidation-susceptible PUFAs, MUFA-rich oils (olive and rapeseed) are less so since MUFAs are much more resistant to peroxidation, and saturated fatty acids (present in coconut oil at levels of ca. 90\% (w/w)) are virtually completely resistant to thermo-oxidation.

Hydroperoxides constitute primary LOPs, and their evolution with increasing heating time is shown in Fig. 3. A comparison of the two types of hydroperoxides amongst the studied culinary oils is shown in Fig. S2. $\dagger$ Whilst both $(E, E)$ and $(Z, E)$-hydroperoxides were detected in all oil types, their signal intensities increased as a function of heating time until $210 \mathrm{~min}$, where a gradual disappearance of the intensity of the hydroperoxides groups was observed. This explains why olive and rapeseed oil spectra show a relatively flatter region in comparison to coconut and sunflower oil (Fig. S2†). This phenomenon was tracked in all studied oils with an illustration shown in Fig. 3. All these primary LOPs (conjugated hydroperoxydienes and hydroxymonoenes, and olefinic resonances of $\alpha, \beta$-UA) have been reported to be present in sunflower oil $(10 \mathrm{~g}$ thermally stressed placed in an oven for $540 \mathrm{~min}$ at $100{ }^{\circ} \mathrm{C}$, and $4320 \mathrm{~min}$ at $70{ }^{\circ} \mathrm{C}$ on a $80 \mathrm{~mm}$ diameter $\times 15 \mathrm{~mm}$ Petri dish), ${ }^{9}$ and corn oils (retained at room temperature in closed receptacles for $121 \mathrm{~min}) .{ }^{10}$ Methanoic acid, which is the simplest carboxylic acid (which arises from the degra- 


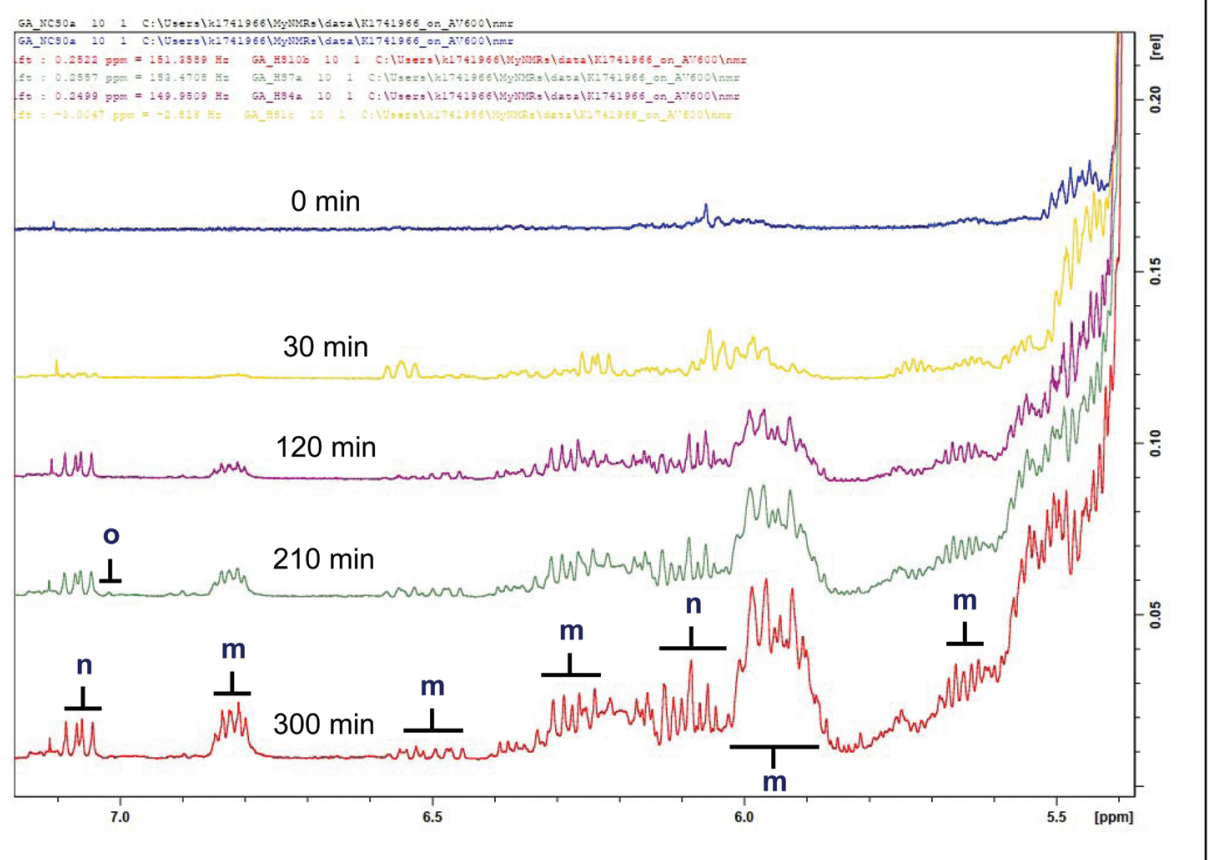

Fig. $2{ }^{1} \mathrm{H}$ NMR spectra of conjugated diene hydroperoxydienes and hydroxymonoenes (primary LOPs), and olefinic resonances of $\alpha, \beta$-unsaturated aldehydes present in the 5.4-7.1 ppm regions of sunflower oil thermally-stressed continuously throughout a $300 \mathrm{~min}$ period. For $\mathrm{m}$ and $\mathrm{n}$ there is overlap, suggesting several unsaturated chain lengths, hence the difference in apparent integral. Abbreviations: $d$, doublet; $t$, triplet; $m$, multiplet; dd, double doublet; CHPDs, conjugated hydroperoxydienes. Letter assignments correspond to those provided in Table 2.

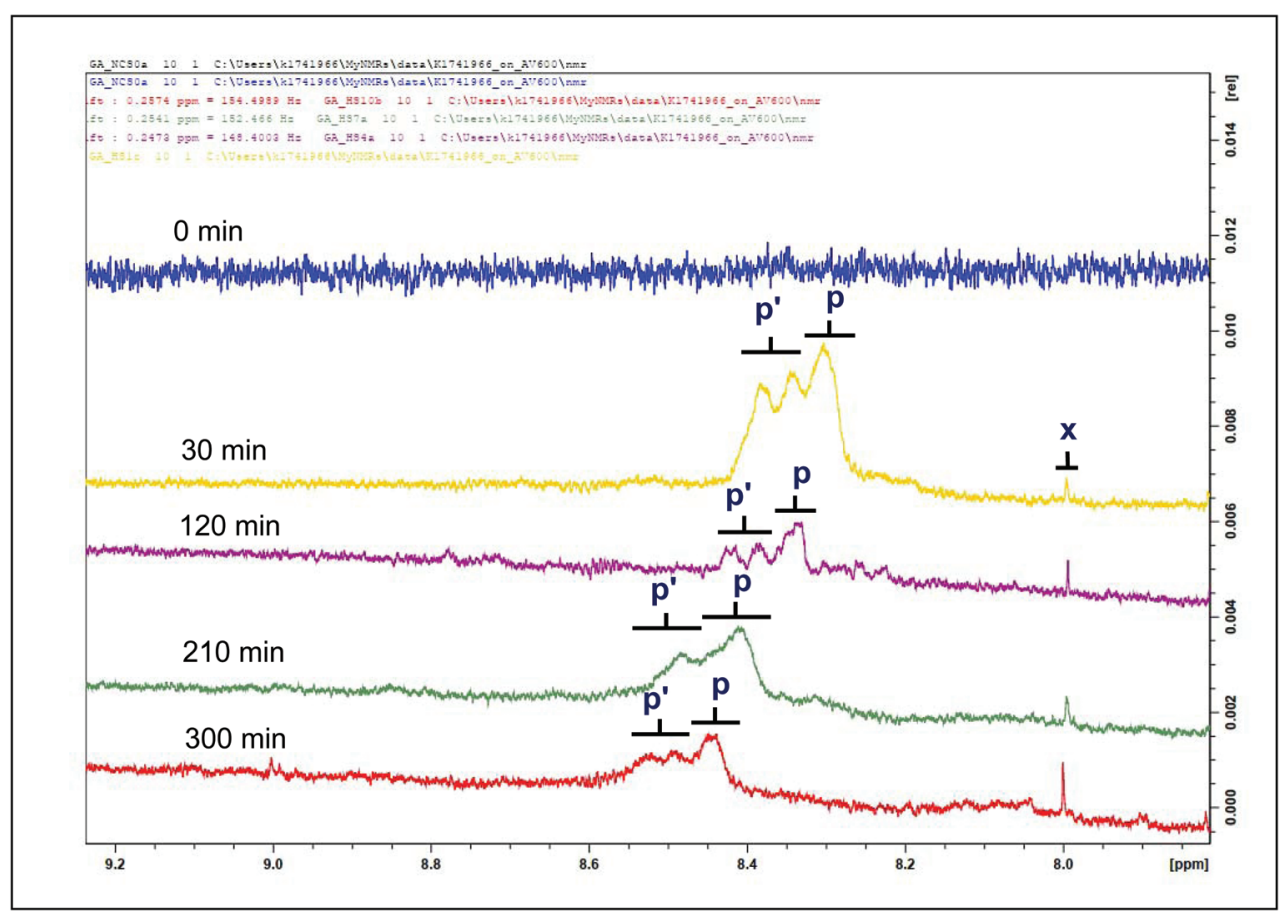

Fig. $3{ }^{1} \mathrm{H}$ NMR spectra of a hydroperoxide group (primary LOPs) and methanoic acid present in the 7.5-9.2 ppm regions of sunflower oil thermally stressed continuously throughout a 300 min period. Signal $p$ is a designated $\mathrm{OOH}-(E, E)$ hydroperoxide function. Signal $\mathrm{p}^{\prime}$ is a designated $\mathrm{OOH}-$ $(Z, E)$ hydroperoxide function. Letter assignments correspond to those provided in Table 2. 


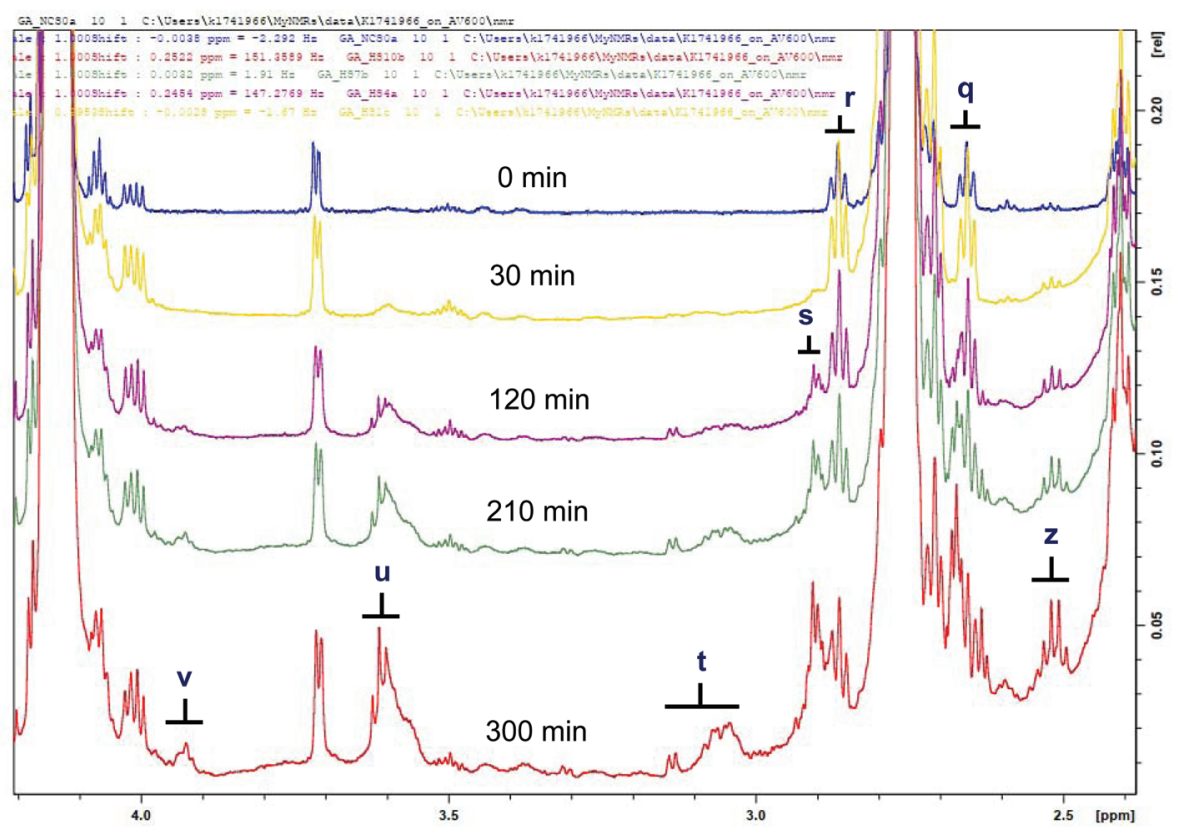

Fig. $4{ }^{1} \mathrm{H}$ NMR spectra of epoxides (secondary LOPs) and primary alcohols present in the 2.4-4.2 ppm regions of sunflower oil thermally stressed continuously throughout a 300 min period. Letter assignments correspond to those in Table 3.

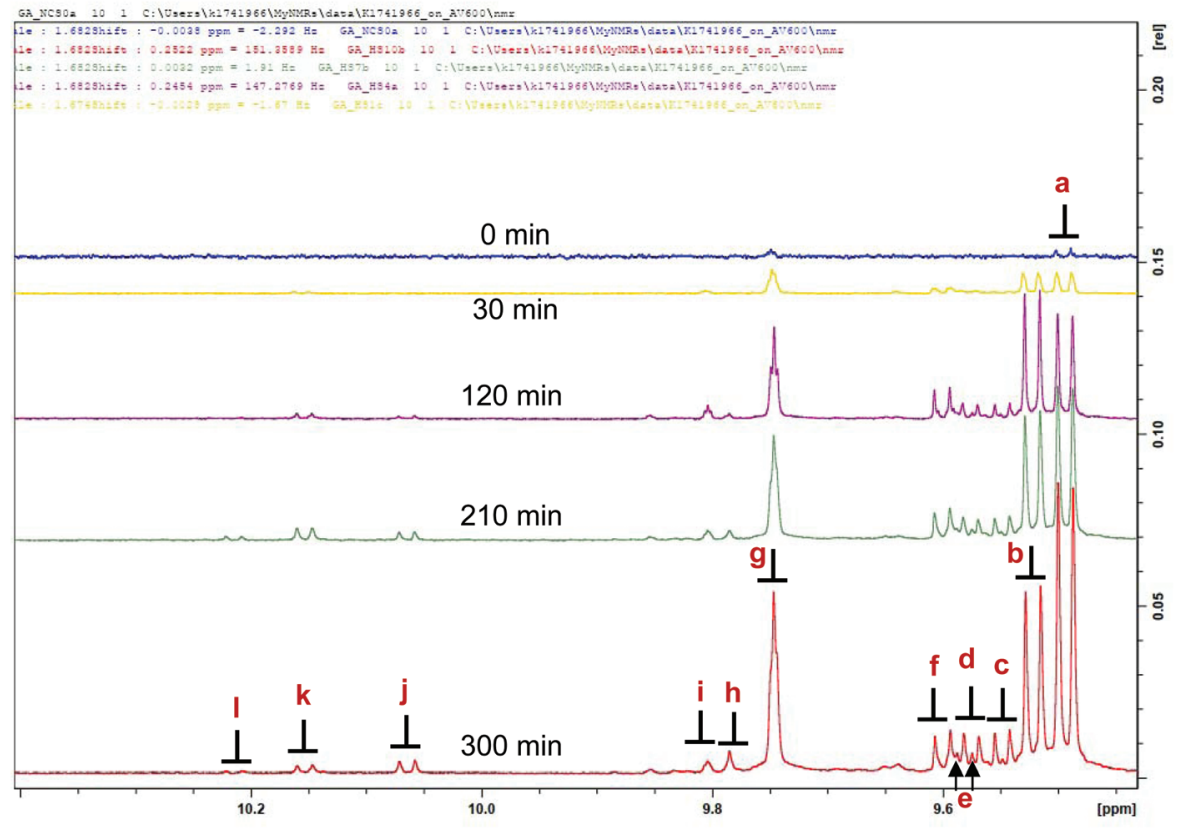

Fig. $5{ }^{1} \mathrm{H}$ NMR spectra of aldehydes (secondary LOPs) present in the 9.3-10.4 ppm regions of sunflower oil thermally stressed continuously over a 300 min period. Letter assignments correspond to those in Table 4.

dation of malondialdehyde, another aldehydic LOP), was also detected in the thermally-stressed oils (Fig. 3 and S2 $\dagger$ ). Being of low toxicity, methanoic acid is used as a food additive.
The evolution of methanoic acid was proportional to the duration of thermal stressing at constant temperature, $180{ }^{\circ} \mathrm{C}$ (Fig. 3 and S2 $\dagger$ ). 


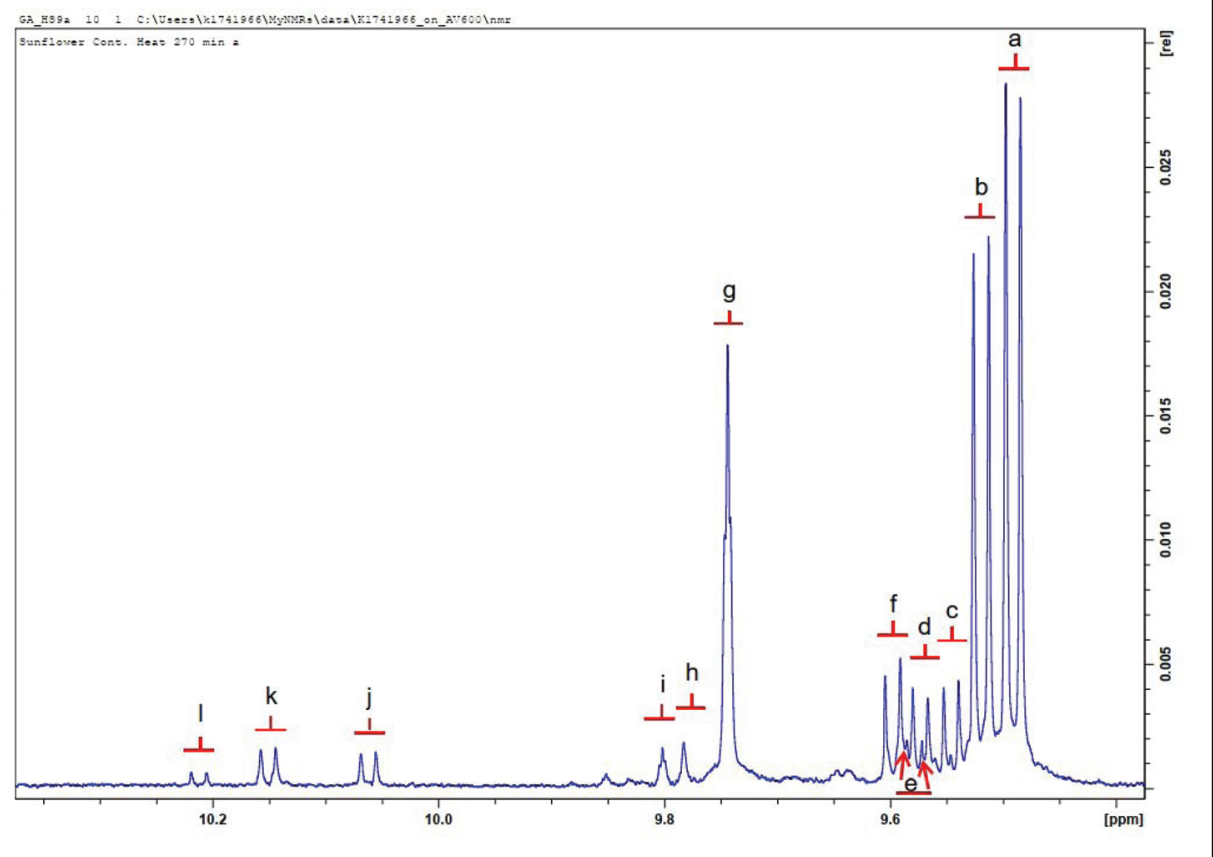

Fig. 6 Expanded aldehydic $-\mathrm{CHO}$ function region $(\delta=9.40-10.36 \mathrm{ppm})$ of ${ }^{1} \mathrm{H}$ NMR spectra of a thermally-stressed sunflower oil. Letters assigned to signals correspond to those in Table 4.

Also classified as secondary LOPs, epoxides and primary alcohols were also detected in the studied oils (Table 3, and Fig. $4, \mathrm{~S} 3 \dagger)$. These epoxides are proposed to be generated from linoleic and oleic acyl groups. ${ }^{6,10,12}$ The high level of distinction in the patterns of these products observed in the studied oils was ascribable to the identification of signals $\mathrm{s}$ and $\mathrm{v}$, namely 9,10-12,13-diepoxyoctadecanoate (predominant in sunflower and rapeseed oils), and those of primary alcohols (predominant in coconut and sunflower oil) (Fig. S3†). Such fatty acid epoxides can cause degeneration and necrosis of leukocytes. They have also been associated with organ malfunction, breast carcinogenesis and cell proliferation. ${ }^{13,14}$ The presence of these LOPs in thermally-stressed culinary oils poses a significant health risk to consumers, and therefore such considerations should to be attended to. Epoxides have also been reported in thermo-oxidized sunflower and extra-virgin olive oils. ${ }^{10,12}$

The aldehydic LOPs discussed in this investigation can be categorised as either $\alpha, \beta$-unsaturated $(\alpha, \beta$-UAs) or saturated aldehydes.

Fig. 5 Shows the ${ }^{1} \mathrm{H}$ NMR aldehydic-CHO function spectral regions demonstrating their evolution during the thermal stressing of culinary oil products.

$\alpha, \beta$-Unsaturated aldehydes are considered more toxic than saturated ones. ${ }^{5,16,17}$ Appearing as doublets in the ${ }^{1} \mathrm{H}$ NMR spectra acquired; signals $a, b, c, d, e, f$ and $j$, classified as $\alpha, \beta$-UAs; were identified as $(E)$-2-alkenals, $(E, E)$-2,4-alkadienals, 4,5-epoxy-(E)-alkenals, 4-hydroxy-(E)-2-alkenals, 4-hydroperoxy(E)-2-alkenals, $(Z, E)$-2,4-alkadienals and (Z)-2-alkenals respectively. ${ }^{6,15}$ Meanwhile, there is a significant level of overlap of the hydroperoxy- and hydroxy-substituted (E)-2-alkenals. Signals $\mathrm{g}, \mathrm{h}$ and i represent those arising from saturated aldehydes which are all triplets with small coupling constants. Signals $\mathrm{g}$ and $\mathrm{i}$ are assignable to $n$-alkanals, with the latter being representative of low-molecular-mass $n$-alkanals, predominantly $n$-propanal and $n$-butanal. ${ }^{6}$ Signal $\mathrm{h}$ is an oxygen-substituted $n$-alkanal class, i.e. 4-oxo- $n$-alkanals. ${ }^{6}$ Doublet signals $\mathrm{k}$ and $\mathrm{l}$ presumably represent alkenal species, probably $(Z)$-isomers from their corresponding $J$ values $(8.08 \mathrm{~Hz}){ }^{15}$

Pioneering studies in lipid oxidation process has made use of several classical qualitative and quantitative standard methods that made important contributions in understanding the mechanisms, dynamics and the evolution of both primary and secondary LOPs. However, these methods, which include peroxide value (PV), conjugated dienes (CDs) and conjugated trienes (CTs), para-anisidine value ( $p \mathrm{AV})$, and thiobarbituric acid-reactive substances (TBARS), have all been criticised as being laborious and lacking in specificity regarding the nature of potential LOPs conceivably analysed, giving rise to inaccurate interpretations and conclusions, and inefficient applications. ${ }^{18,19}$ Indeed, in addition to a marked lack of specificity, the still routinely employed TBARS assay is known to artefactually generate a variety of LOPs, both primary and secondary, during the heating stage (usually for a period of 15 min at $\left.c a .95{ }^{\circ} \mathrm{C}\right) .{ }^{5}$ On the contrary, one-dimensional $\left({ }^{1} \mathrm{H}\right)$ and two-dimensional $\left({ }^{1} \mathrm{H}^{-1} \mathrm{H}\right.$ and $\left.{ }^{1} \mathrm{H}^{13} \mathrm{C}\right)$ nuclear magnetic resonance (NMR) analyses are established techniques that are faster and dependable with regard to the identification and determination of variable concentrations of cytotoxic and gen- 
otoxic LOPs in food lipid systems. ${ }^{5,6}$ The value of the NMR technique is further exemplified by the experimental applications of Pure Shift Yielded by Cherp Excitation (PSYCHE) and hyphenated diffusion techniques (PSYCHEiDOSY) for the resolution of small molecule resonances in complex multicomponent mixtures. ${ }^{20}$ Nonetheless, primary oxidation products experimentally determined as PV and CD values have been shown to positively correlated with lipid hydroperoxide levels determined by the ${ }^{1} \mathrm{H}$ NMR technique. ${ }^{21}$

This research will therefore explore the of molecular 'patterns' of toxic LOPs generated in a wide range of culinary oil or fat products, particularly when exposed to thermal stressing episodes performed according to standard frying practices.

\section{Materials and methods}

\subsection{Preparation of oil samples}

Culinary oils were procured from a local supermarket outlet in Manchester, United Kingdom. These were: 100\% organic virgin coconut oil $(90.22 \%(\mathrm{w} / \mathrm{w})$ SFAs, with unspecified amounts of MUFAs and PUFAs); olive oil (15.02\% (w/w) SFAs, $76.38 \%(\mathrm{w} / \mathrm{w})$ MUFAs, and $8.60 \%(\mathrm{w} / \mathrm{w})$ PUFAs); rapeseed oil (7.96\% (w/w) SFAs, 63.03\% (w/w) MUFAs, and 29.01\% (w/w) PUFAs); and sunflower oil (10.87\% (w/w) SFAs, $28.26 \%(w / w)$ MUFAs, and $60.87 \%$ (w/w) PUFAs). Based on the percentage FA compositions originally printed on their labels (values in parenthesis) as purchased, coconut oil is classified as SFA-rich, olive and rapeseed as MUFA-rich, and sunflower oil as PUFArich oils. However, it should also be noted that rapeseed oil has a much higher level of the omega-3 PUFA linolenic acid (predominantly as triacylglycerols), i.e. approximately $10 \%$ (w/w); linolenoylglycerols are, of course, more susceptible to peroxidation than linoleoylglycerols, and give rise to a different pattern of secondary aldehydic LOPs than those derived from the latter source.

\subsection{Thermal stressing of culinary oil samples}

Thermal stressing of coconut, olive, rapeseed and sunflower oils was conducted in the presence of atmospheric oxygen at $180^{\circ} \mathrm{C}$ with increasing time periods in three different series of standard heating or cooking practices. For comparative, quantitative ${ }^{1} \mathrm{H}$ NMR evaluations, it was ensured that the same electronic balance $( \pm 0.1 \mathrm{~g}$ accuracy, Mettler (UK), Model AT261) and electronically-controlled hot-plate $(230 \mathrm{~V}, 50 \mathrm{~Hz}, 750 \mathrm{~W}$; Stuart heat-stir (UK), Model SB162) were used for the weighing and heating experiments respectively. All glassware was dried under $\mathrm{N}_{2}$ and completely sealed after discharging the amount required for heating.

\subsection{Continuous heating episodes}

The first type of heating, termed "continuous" heating, and initially described in ref. 22 was adapted and slightly modified: $20 \mathrm{~g}$ of oil samples were weighed directly into $100 \mathrm{~mL}$ beakers of lipid-air surface area $70.69 \mathrm{~cm}^{2}$, and then were heated on an electronically-controlled hot-plate operated at $180^{\circ} \mathrm{C}$ for a total duration of $300 \mathrm{~min}$. With the aid of a $230 \mathrm{~mm}$ Fischerbrand glass Pasteur pipette, $1.0 \mathrm{~mL}$ of thermally-stressed oil was sampled from the hot oil source into glass vials at regular $30 \mathrm{~min}$ time intervals for a total period of $300 \mathrm{~min}$ (as shown in Fig. 7). Cooling of the sampled oils was conducted rapidly on ice, and they were then processed for rapid ${ }^{1} \mathrm{H}$ NMR measurements. The heating experiment and sampling were repeated in triplicate for each oil evaluated in this manner. Control experiments for the studied culinary oils were their respective unheated samples. The results of all detected, quantified LOPs were presented as mean \pm standard deviation (SD) values.

\subsection{Discontinuous heating and cooling episodes}

In the discontinuous heating and cooling series of experiments, the same process outlined in section 2.3 above was followed. These were then heated in a discontinuous fashion for 30 min heating on an electronically-controlled hot-plate plate operated at $180{ }^{\circ} \mathrm{C}$. This was followed by an ambient temperature cooling period of $120 \mathrm{~min}$ (Fig. 8). An aliquot of $1.0 \mathrm{~mL}$ of thermally-stressed oil was sampled at specified times (shown as in Fig. 4) from the thermally-oxidised oil into glass vials for ${ }^{1} \mathrm{H}$ NMR analysis. Sampled oils were then rapidly cooled on ice. The discontinuous heating and cooling experiment was conducted in triplicate for each culinary oil. Unheated culinary oil samples served as essential controls. All LOP levels reported were expressed as mean \pm SD values.

\section{$2.5{ }^{1} \mathrm{H}$ NMR analysis of LOPs}

Changes in the composition of all examined oils were monitored by ${ }^{1} \mathrm{H}$ NMR analysis conducted on a Bruker Avance III $400 \mathrm{MHz}$ (BBI) and $600 \mathrm{MHz}$ (TXI) spectrometers (Kingston University, London) operating at 400.13 and $600.13 \mathrm{MHz}$ frequencies respectively, and a probe temperature of 298 K. For both instruments, the following acquisition parameters were employed: size of fid 65 536; number of scans 256; probe temperature $300 \mathrm{~K}$; spectral width $20.573 \mathrm{ppm}$; relaxation delay $1.000 \mathrm{~s}$; acquisition time $4.819 \mathrm{~s}$; pulse width $90{ }^{\circ} \mathrm{C}$; and total acquisition time $16 \mathrm{~min}$.

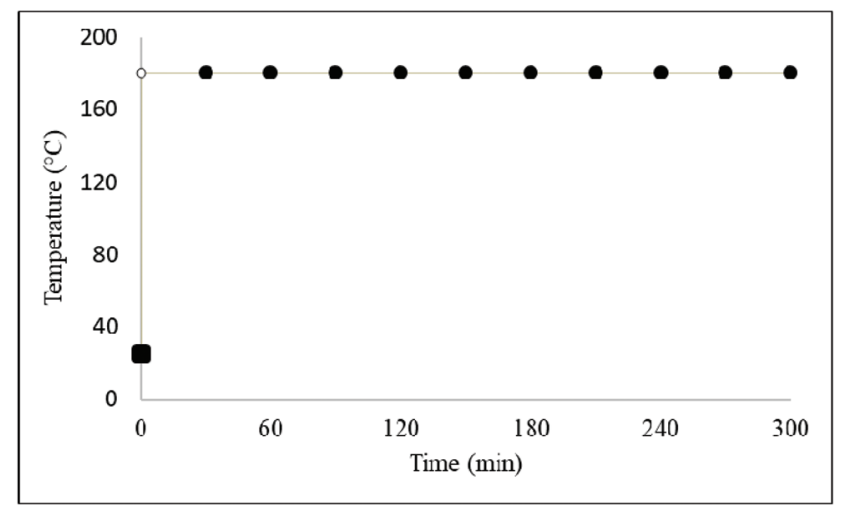

Fig. 7 A continuous heating assay and timely sampling method for thermally-stressed culinary oils. 


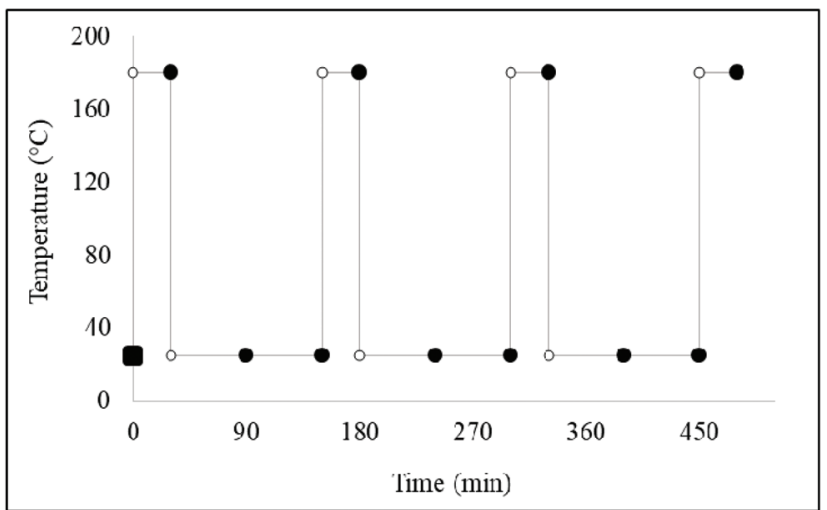

Fig. 8 A discontinuous heating and intermittent cooling assay involving a timely sampling method for each thermally-stressed culinary oil investigated.

An $0.30 \mathrm{~mL}$ aliquot of each control (unheated) and thermally-stressed culinary oil was diluted with a $0.60 \mathrm{~mL}$ volume of deuterated chloroform $\left(\mathrm{CDCl}_{3}\right)$ (99.8\% purity, Sigma-Aldrich Chemical Co., UK). $\mathrm{CDCl}_{3}$, which served to provide a field frequency lock for the studied oil samples, had a chemical shift $(\delta)$ value of $7.283 \mathrm{ppm}$. An aliquot $(0.50 \mathrm{~mL})$ of the resulting lipid- $\mathrm{CDCl}_{3}$ mixture was then pipetted into a $5 \mathrm{~mm}$ diameter NMR tube (Norrell HT, GPE Scientific). This was further treated with a $0.10 \mathrm{~mL}$ of a solution of 1,3,5-tribromobenzene (Sigma-Aldrich) (TBB) (prepared by dissolving $4.14 \mathrm{mg}$ TBB in $2.0 \mathrm{~mL}$ of $\mathrm{CDCl}_{3}$ ). With a $7.537 \mathrm{ppm} \delta$ value, TBB served as an internal quantitative NMR standard in the determination of NMR-detectable peroxidation products in thermally-stressed oil samples. All ${ }^{1} \mathrm{H}$ NMR chemical shifts, regardless of oil nature, were referenced to tetramethylsilane (TMS) $(\delta=$ $0.000 \mathrm{ppm})$ and/or residual chloroform $(\delta=7.283 \mathrm{ppm})$. The ${ }^{1} \mathrm{H}$ NMR spectra obtained in this study were analysed using Topspin 3.5 sp7 (Bruker Biospin), and were found to be fully consistent with literature reports. ${ }^{11,21,23,24}$

\subsection{Analysis of culinary oil LOPs}

Lipid oxidation products (LOPs) were identified from ${ }^{1} \mathrm{H}$ NMR spectra acquired in the present study with the aid of literature references. ${ }^{6,23,24}$ In addition to $1 \mathrm{D}{ }^{1} \mathrm{H}$ NMR analysis, carbon-13 $\left({ }^{13} \mathrm{C}\right)$ and ${ }^{13} \mathrm{C}$ Distortionless Enhancement by Polarization Transfer $\left({ }^{13} \mathrm{C}\right.$ DEPT-90) spectra, together with other two-dimensional (2D) NMR spectroscopic analyses, i.e. Heteronuclear Single Quantum Correlation (HSQC) and Correlation Spectroscopy (COSY) were also employed to confirm the molecular identification of LOPs.

\subsection{Experimental design and statistical analysis}

For experiments involving the exposure of CLO products to thermal stressing episodes, the experimental design for univariate analysis of the ${ }^{1} \mathrm{H}$ NMR aldehyde classification concentration datasets involved an analysis-of-covariance (ANCOVA) model, which incorporated 3 prime factors, and a total of 7 sources of variation: (1) that 'between-oil products', qualitative fixed effect $\left(\mathrm{O}_{i}\right)$; (2) that 'between-sampling time-points' quantitative fixed effect 'nested' within the culinary oil classifications $\left(\mathrm{T}_{j}\right)$; (3) the thermal stressing method employed, i.e. continuous versus discontinuous heating strategies $\left(\mathrm{M}_{k}\right) ;(4)$ the oil classification $\times$ sampling time-point first-order interaction effect $\left(\mathrm{OT}_{i j}\right)$; (5) the oil classification $\times$ thermal stressing method first-order interaction effect $\left(\mathrm{OM}_{i k}\right)$; $(6)$ the sampling time-point $\times$ thermal stressing method interaction effect $\left(\mathrm{TM}_{j k}\right)$; (7) the 'between-replicate' random effect nested within factors, $R_{(i j k) l}$ (1), (2) and (3). The mathematical model for this experimental design is shown eqn (1), in which $y_{i j k l m}$ represents the (univariate) aldehyde ISB dependent variable values observed, $\mu$ its overall population mean value in the absence of any significant, influential sources of variation, and $e_{i j k l m}$ the unexplained error (residual) contribution.

$$
y_{i j k l}=\mu+\mathrm{O}_{i}+\mathrm{T}_{j}+\mathrm{M}_{k}+\mathrm{OT}_{i j}+\mathrm{OM}_{\mathrm{jk}}+\mathrm{TM}_{j k}+R_{(i j k) l}+e_{i j k l}
$$

ANCOVA was conducted with XLSTAT2016 software (Addinsoft, Paris, France). Post-hoc analysis of significant differences observed between individual CLO products and sampling time-points were performed using Tukey's test.

Further analysis of the univariate aldehydic LOP concentration dataset was performed by comparisons of their least square mean (LSM) values. For example, LSM values for the 'between-thermal stressing method' factor were computed by adjusting for the major 'between oil classifications' and 'between-sampling time-point' sources of variation. The statistical significance of these LSM differences were determined by Tukey's ANCOVA post-hoc test.

\section{$2.8 \quad{ }^{1} \mathrm{H}$ NMR analysis of aldehydes in fried potato chip servings}

For domestic ('at-home') deep-frying episode experiments, batches of hand-cut chips of lengths and widths of $87.00 \pm$ 1.15 and $12.70 \pm 0.36 \mathrm{~mm}$ (mean $\pm \mathrm{SEM}$ ) respectively were consecutively fried 8 times daily for two days, and then only 3 times on the third day using sunflower oil. The deep-frying facility employed was a domestic model (EasyPro, Tefal) equipped with a variable thermostat and an inert cross-lined steel mesh for the purpose of lowering the chips into the oil without contacting the fryer's inner surface. This deep fryer was filled with 3.00 litres of oil according to the manufacturer's instructions, and $400 \pm 10 \mathrm{~g}$ of potato chips then deep fried at a temperature of $170{ }^{\circ} \mathrm{C}$ for a period of $10.0 \mathrm{~min}$.

The $\%(w / w)$ total PUFA, MUFA and SFA contents of the sunflower oil employed in these studies was 61.0, 28.0 and $11.0 \%(\mathrm{w} / \mathrm{w})$ respectively.

Each frying oil used was allowed to cool for a period of exactly $30 \mathrm{~min}$ between each repetitive frying episode (a total of 8 per full daily cycle, as noted above). Following each 10 min frying episode, chips were thoroughly shaken in their wire basket for $15 \mathrm{~s}$, and then allowed to drain therein for $30 \mathrm{~s}$ to remove excess oil. Chips were then transferred to a steel mesh draining board. 
At the final collection time-point on day 3 (corresponding to the $19^{\text {th }}$ frying session), 2 randomly-selected samples of chips were transferred to plastic-stoppered sample tubes and immediately frozen at a temperature of $-20{ }^{\circ} \mathrm{C}$ until transported to the laboratory where they were then stored at $-80{ }^{\circ} \mathrm{C}$ for a maximum duration of $18 \mathrm{~h}$ prior to ${ }^{1} \mathrm{H}$ NMR analysis. On completion this finalised frying episode, duplicate samples of sunflower oil were also collected for analysis, and these were also stored prior to analysis in the same manner as the potato chip samples, as were duplicate unheated (control) frying oil samples. Two samples of the unfried potatoes were also collected and stored in this manner.

\section{Results and discussion}

\subsection{Culinary oil FA compositions and IV}

The molar percentages and iodine value of a series of FA acyl groups were deduced from the ${ }^{1} \mathrm{H}$ NMR spectral profiles of the oil samples investigated. These deductions were based on the

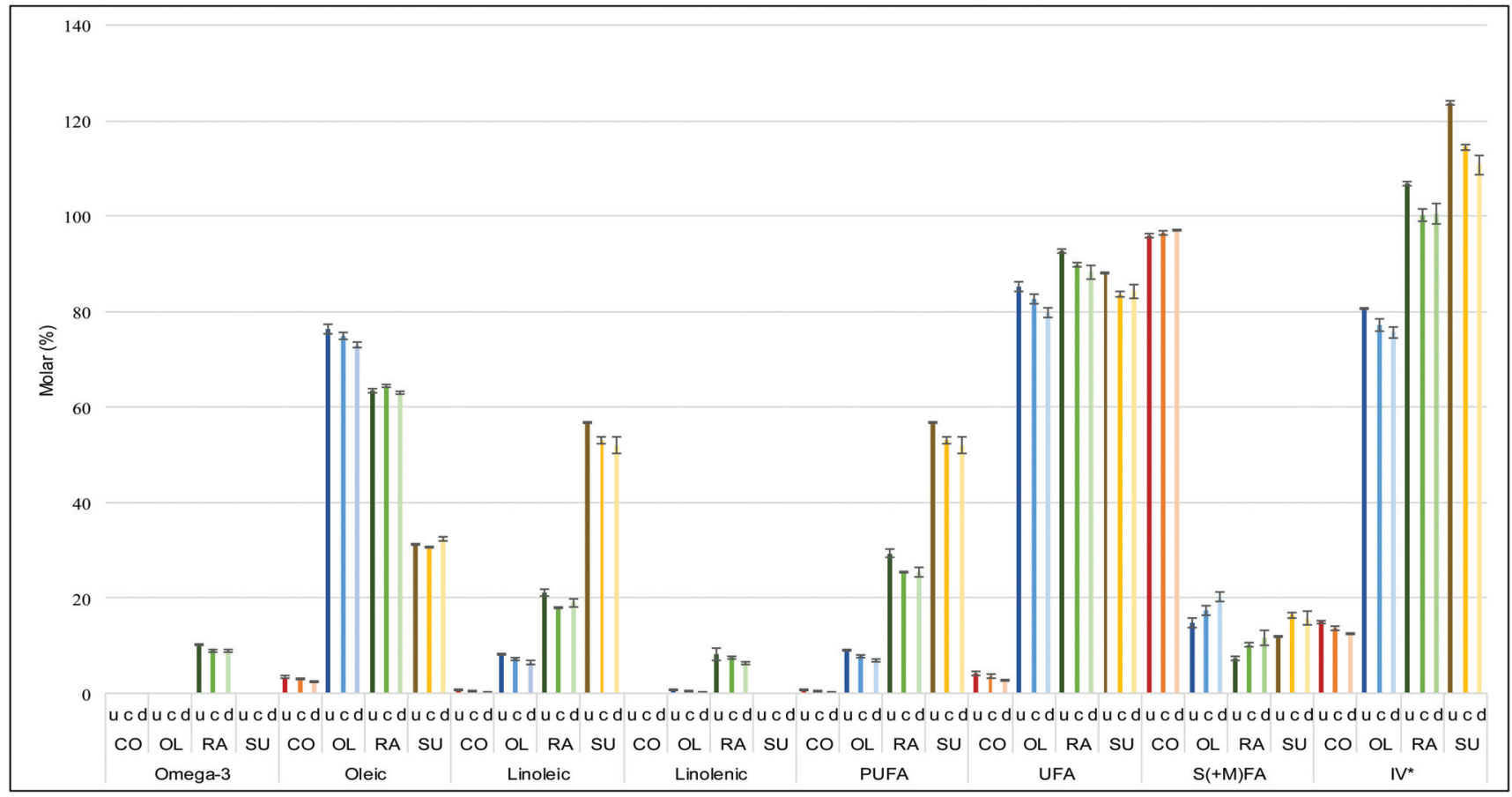

Fig. 9 Concentrations of acyl groups at a heating time-point of $120 \mathrm{~min}$ for continuous and discontinuous heating systems involved in the thermal stressing of coconut, olive, rapeseed and sunflower oils. Abbreviations: Unheated oil (u), Continuous heating (c), Discontinuous heating and cooling (d), Coconut oil (CO), Olive oil (OL), Rapeseed oil (RA), Sunflower oil (SU) and lodine value (IV). S(+M)FA implies saturated and modified fatty acyl groups which is only applicable after thermal stressing of culinary oil. *lodine value is expressed as unit (right axis).

Table 4 Assignments of $-\mathrm{CHO}$ functional groups of ${ }^{1} \mathrm{H}$ NMR signals of secondary aldehydic LOPs present in the ${ }^{1} \mathrm{H}$ NMR profiles of sunflower oil thermally stressed continuously throughout a 300 min period. Chemical shift, multiplicities and associated -CHO function identities are given

\begin{tabular}{|c|c|c|c|c|}
\hline Signal & Chemical shift (ppm) & Multiplicity & \multicolumn{2}{|l|}{ Functional group } \\
\hline a & 9.472-9.505 & $\mathrm{d}$ & $-\mathrm{CHO}$ & $(E)$-2-Alkenals \\
\hline c & $9.535-9.557$ & $\mathrm{~d}$ & $-\mathrm{CHO}$ & 4,5-Epoxy- $(E)$-alkenals \\
\hline d & $9.557-9.583$ & $\mathrm{~d}$ & $-\mathrm{CHO}$ & 4-Hydroxy- $(E)-2$-alkenals \\
\hline e & $9.570-9.587$ & $\mathrm{~d}$ & $-\mathrm{CHO}$ & 4-Hydroperoxy- $(E)$-2-alkenals \\
\hline $\mathrm{h}$ & $9.776-9.793$ & $\mathrm{t}$ & $-\mathrm{CHO}$ & 4-Oxo-alkanals \\
\hline i & $9.793-9.809$ & $\mathrm{t}$ & $-\mathrm{CHO}$ & $n$-Alkanals of low-molecular-mass (propanal and butanal) \\
\hline j & $10.048-10.075$ & $\mathrm{~d}$ & $-\mathrm{CHO}$ & $(Z)$-2-Alkenals ${ }^{a}$ \\
\hline $\mathrm{k}$ & $10.138-10.163$ & $\mathrm{~d}$ & $-\mathrm{CHO}$ & Unidentified unsaturated aldehyde \\
\hline 1 & $10.201-10.225$ & $\mathrm{~d}$ & $-\mathrm{CHO}$ & Unidentified unsaturated aldehyde \\
\hline
\end{tabular}

Abbreviation: d, doublet; t, triplet. ${ }^{a}$ First identified by ref. 15. Letter assignments correspond to those in Fig. 5 and 6. 
assumption that the area of the signals generated in each ${ }^{1} \mathrm{H}$ NMR spectrum is proportional to the number of ${ }^{1} \mathrm{H}$ nuclei giving rise to them, and that the proportionality constant is the same in all cases. Acyl groups such as omega-3, oleic (or monounsaturated), linoleic and linolenic (or polyunsaturated), as well as saturated and modified groups were inferred from the ${ }^{1} \mathrm{H}$ NMR spectra acquired (Fig. 1). PUFAs, as well as total unsaturated acyl groups, were calculated from the data generated from the mathematical computations of oleic, linoleic and linolenic acyl groups (ESI Summary S1 $\dagger$ ). The determination of iodine value (IV) was deduced directly from the olefinic protons observed in the ${ }^{1} \mathrm{H}$ NMR spectra of culinary oils6 (ESI section S1 $\dagger$ ). All deductions of the acyl group compositions were computed in triplicate, and the results obtained are provided as mean \pm SD values (Table 3). ${ }^{6,25}$

3.1.1 Continuous heating. The changes of the key FA acyl groups of culinary oils monitored throughout the processing time, under continuous heating conditions can be observed in the ESI (Fig. S5 $\dagger$ ). As noted above, the reduction in unsaturated acyl groups, in response to thermo-oxidation exposure time, demonstrates the increment and development of saturated and modified acyl groups, as well as those of LOPs. Linoleoylglycerols (all oils investigated) and linolenoylglycerols (rapeseed oil) decreased appreciably in terms of their molecular percentages over a 300 min duration of continuous heating. The differences in the degree of unsaturation of the culinary lipids influenced the level of changes in the acyl groups. Oleic acyl groups showed a unique trend, since their levels stabilized in rapeseed oil, increased somewhat in sunflower oil, and decreased significantly in coconut and olive oils. This explains the dynamism of thermo-oxidative resistance of MUFA acylglycerols, as well as the susceptibility of PUFAs to thermo-oxidation. Cumulatively, the total unsaturated acyl groups, specifically UFAs, decreased from 4.1 to $1.8 \%, 85.2$ to $74.2 \%, 92.7$ to $82.2 \%$, and 88.1 to $75.7 \%$ in coconut, olive, rapeseed and sunflower oils, respectively. Simultaneously, the saturated and modified acyl $(\mathrm{S}(+\mathrm{M}) \mathrm{FA})$ groups increased from 95.9 to $98.2 \%, 14.8$ to $25.7 \%, 7.3$ to $17.7 \%$, and 11.9 to $24.3 \%$ in coconut, olive, rapeseed and sunflower oils, respectively (Fig. S5 in ESI $\dagger$ ). The percentage loss of UFA (and corresponding gain in SFA) groups of the culinary lipids was in the order sunflower oil $(12.4 \%)>$ olive and rapeseed oil (10.9\% and $10.4 \%$, respectively) $>$ coconut oil $(2.3 \%)$. At the 300 min time-point, IV had also decreased from 15.0 to 11.8 units (coconut oil), 80.6 to 68.2 units (olive oil), 106.8 to 89.1 units (rapeseed oil) and 123.7 to 99.9 units (sunflower oil) (data not shown). Overall, changes in acyl groups were therefore small in coconut oil, in view of its much lower degree of unsaturation. The magnitude of these changes increased with increasing unsaturation degree of the oils explored here, as expected. However, it should also be noted that as virgin coconut oil has a smoke-point below the standard frying temperature of $180{ }^{\circ} \mathrm{C}$, and hence would be expected to degrade markedly at this temperature (Fig. 9).

3.1.2 Discontinuous heating and cooling experiments. Modifications in the acyl groups of coconut, olive, rapeseed

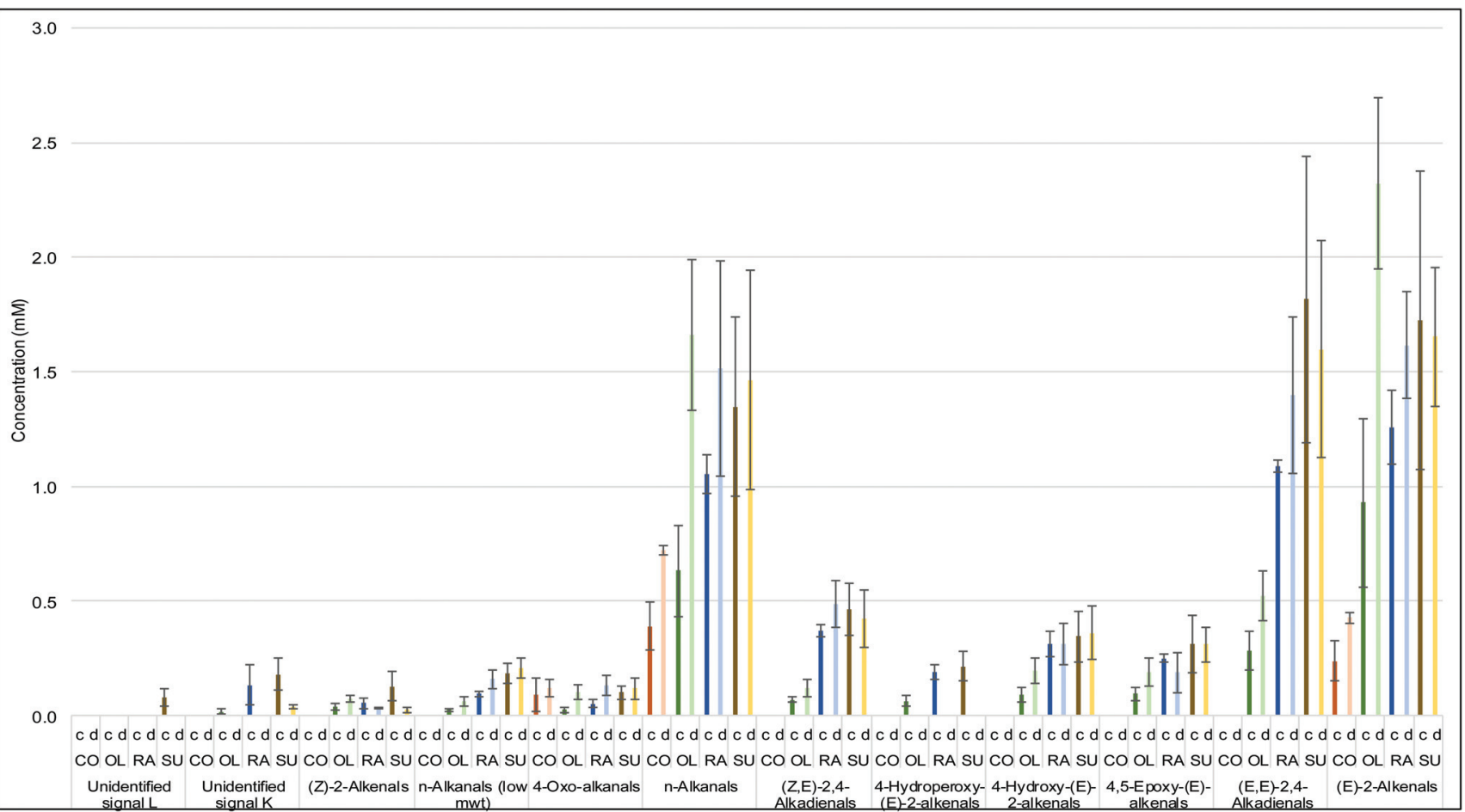

Fig. 10 Concentrations of LOPs at the 120 min time-point comparing continuous and discontinuous heating systems of thermally-stressed coconut, olive, rapeseed and sunflower oils. Abbreviations: Continuous heating (c), Discontinuous heating and cooling (d), Coconut oil (CF), Olive oil (OL), Rapeseed oil (RA), Sunflower oil (SU). 
and sunflower oils when subjected to a discontinuous heating and cooling system are provided in the ESI (Fig. S6 $\dagger$ ). The cycle of $30 \mathrm{~min}$ heating with an intermittent 120 min cooling duration influenced the characteristics of the oils' acyl functions. The degradation of unsaturated FA acyl groups was met with a simultaneous build-up of saturated and modified ones on the completion of every $30 \mathrm{~min}$ heating cycle involved. However, the percentage distribution of all acyl groups remained unchanged during the 120 min intermittent cooling period. This experiment demonstrates that in the absence of heat, which promotes the autocatalytic peroxidation of UFAs (ESI Fig. S2 and S3†), the rate of oxidation is low (as expected). Unsaturated acyl group contents, specifically UFAs, decreased from 4.1 to $2.9,85.2$ to $79.8,92.7$ to 88.8 , and 88.1 to 84.3 molar \% in coconut, olive, rapeseed and sunflower oils, respectively. This was simultaneously accompanied by increases in saturated and modified acyl groups, specifically S(+M)FA, from 95.9 to $97.1,14.8$ to $20.2,7.3$ to 11.7 , and 11.9 to 15.7 molar \% in coconut, olive, rapeseed and sunflower oils, respectively. In all cases, the percentage loss of UFAs (or gain in $\mathrm{S}(+\mathrm{M}) \mathrm{FA})$ groups was in the order olive $(5.4 \%)>$ rapeseed $(4.3 \%)>$ sunflower $(3.8 \%)>$ coconut oils $(1.2 \%)$. Despite this trend being dissimilar, both continuous and discontinuous systems presented coconut oil as having the least percentage loss of acyl unsaturated FA groups. Similar to continuous heating, IV was also observed at the $450 \mathrm{~min}$ time-point to decrease from 15.0 to 12.5 units (coconut oil), 80.6 to 75.8 units (olive oil), 106.8 to 100.5 units (rapeseed oil) and 123.7 to 110.7 units (sunflower oil) in discontinuous heating episodes (data not shown). Comparatively, percentage losses of acyl groups for all these oil products were substantially lower in the discontinuous than they were in the continuous system. This indicates that the discontinuous model of heating culinary oil acylglycerols may render them safer for human consumption than the continuous model. Coconut oil contained the highest percentage of SFA groups and the lowest levels of oleic, linoleic, PUFA and total UFA groups. Coconut oil therefore exhibited the most heat resistivity in this study. Of course, concentrations of oleoylglycerol acyl groups were in the order olive $>$ rapeseed $>$ sunflower $>$ coconut oil.

Amongst the unsaturated FA-rich oils, the greater the amount of oleic acid, the more resistant the oil to thermo-oxidation, ${ }^{15}$ although SFA's are considerably more resistant to oxidation than MUFAs. Concentrations of linoleic acyl groups and PUFA groups were in the order sunflower oil $>$ rapeseed oil $>$ olive oil > coconut fat. By implication, sunflower oil was most susceptible to thermodegradation. ${ }^{15,26,27}$ Amongst the heating systems employed, discontinuous heating episodes, regardless of the culinary oil tested, had the least impact on preoxidation of oil UFAs. Plots comparing individual FA components are provided in the ESI (Table S1 and Fig. S5, S6†).

\subsection{Evolution of LOPs}

A proposed integrated reaction scheme showing how oxidation products could evolve was adapted from ref. 28 and slightly modified (ESI Fig. S9†). At the pre-heating time-point

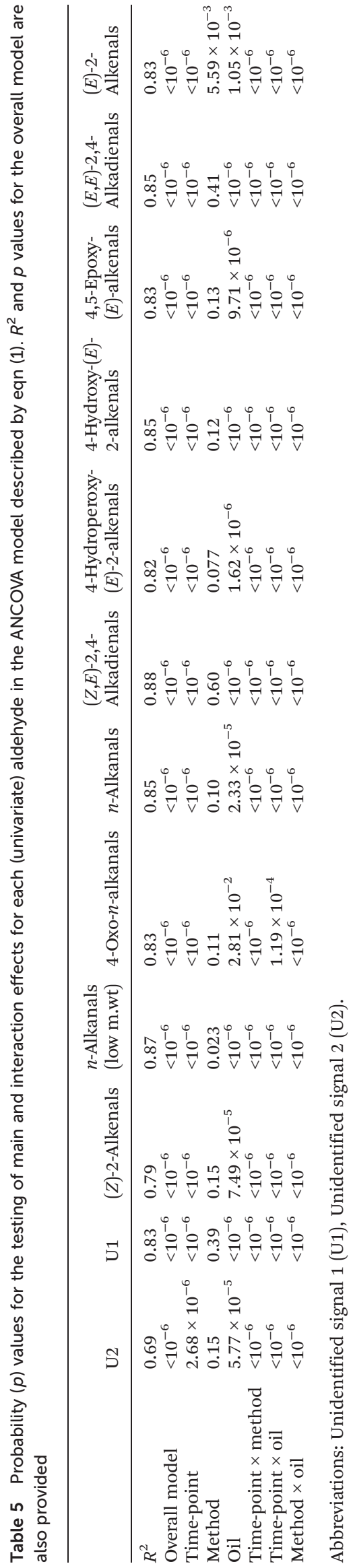


(0.0 min), only very low levels of LOPs are expected to be present. This was correct for coconut and rapeseed oils. However, unheated olive oil was found to contain relatively low levels of $n$-alkanals, $(E, E)$-2,4-alkadienals and $(E)$-2-alkenals at concentrations of $0.09,0.02$ and $0.09 \mathrm{mM}$, respectively. Also, $n$-alkanals $(0.19 \mathrm{mM})$ and $(E)$-2-alkenals $(0.15 \mathrm{mM})$ were quantifiable in unheated sunflower oil. Prior to the ${ }^{1} \mathrm{H}$ NMR measurements, all culinary oils purchased from the local supermarket were stored in dark for not more than 72 hours.

As noted above, $(E, E)$-2,4-alkadienals and $(E)$-2-alkenals are both examples of $\alpha, \beta$-UAs, and therefore their presence in unheated culinary oil presents a potential health risk to consumers. The presence of LOPs in unheated olive and sunflower oils may be attributed to possible exposure of the oils to variable forms of oxidative stress, through industrial refinement and/or subjection to prolonged storage conditions. ${ }^{5,22,29}$

The differences in unsaturation degree account for the proportional distribution of aldehydic LOPs between sunflower and olive oil at time 0 min. ${ }^{5}$ For example, $(E, E)-2,4$-alkadienals, along with 4,5-epoxy-(E)-2-alkenals derived directly therefrom, arise from the peroxidation of PUFAs, whereas $(E)$-2-alkenals and higher molecular mass $n$-alkanals are generated from both MUFAs and PUFAs. Moreover, acrolein, malondialdehyde (MDA) and propanal are generated from omega-3 FAs, almost exclusively from linolenoylglycerols present in vegetable oils, which is particularly notable for rapeseed oil included in the study performed here.

A general summary of observations and rationale for our quantification strategy is provided in the ESI (Summary S2) $\dagger$

The formation of LOPs and the increases in their concentrations in culinary oils throughout our thermo-oxidation processes can be illustrated in Table 4 and Fig. 6 . The intensity of the signals of LOPs generated in the ${ }^{1} \mathrm{H}$ NMR spectra of thermally-stressed culinary oils proportionately correspond to the concentrations of these oxidation products. At a constant temperature of $180{ }^{\circ} \mathrm{C}$, the amounts of LOPs generated increase with time as previously reported. ${ }^{26}$ Also, the degree of unsaturation had a high level of influence on the evolution of LOPs, as expected. ${ }^{4}$ By implication, thermally-stressed SFA-rich coconut oil yielded only three types of LOPs, specifically $(E)-2-$ alkenals (signal a), $n$-alkanals (signal g) and 4-oxo- $n$-alkanals (signal h). However, the concentrations of these LOPs were all lower in comparison to those of the other, much more MUFAand/or PUFA-rich oils tested, as expected from its very high SFA content. There were, however, striking similarities between olive oil and rapeseed oil, since both oils are MUFArich and therefore share a similar degree of thermo-oxidation resistivity characteristics. Sunflower oil yielded the largest proportion of LOPs over a total $300 \mathrm{~min}$ heating period (please refer to ESI Table 2 and Fig. S7, S8 $\dagger$ ). This included the unsaturated aldehyde doublet $\mathrm{k}$ which was absent from the ${ }^{1} \mathrm{H}$ NMR profiles of thermally-stressed coconut oil, as well as those of olive and rapeseed oil. The susceptibility of sunflower oil to thermo-oxidation is certainly attributable to its high content of PUFAs.
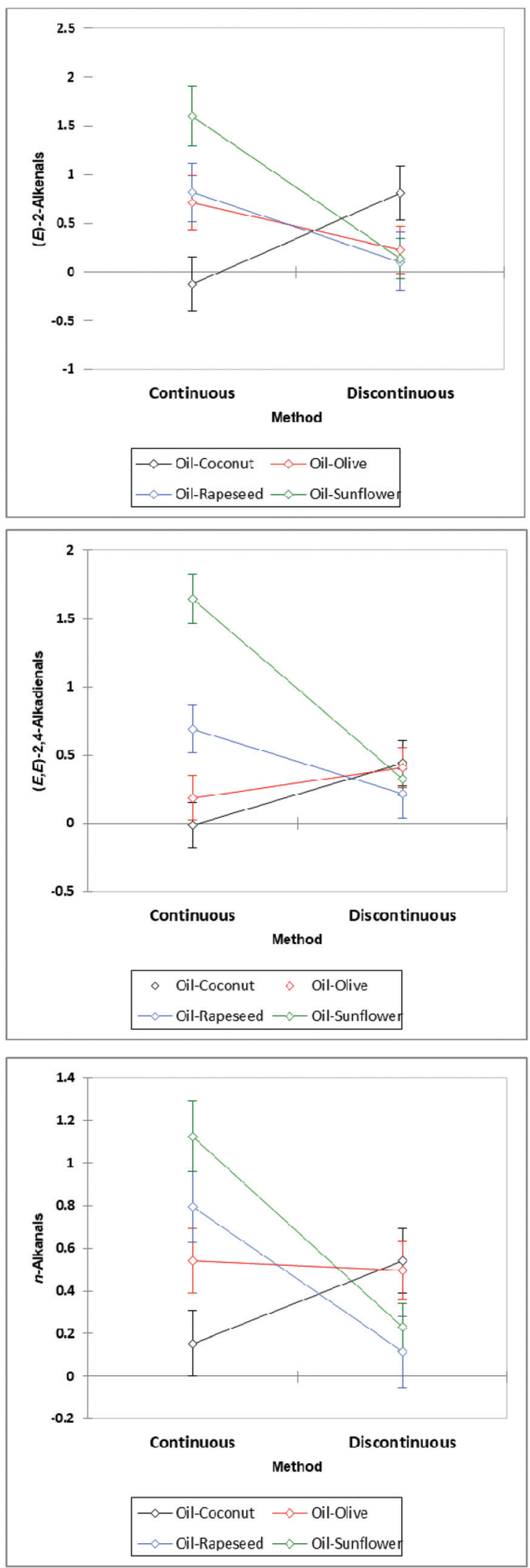

Fig. 11 Plots of LSM values for (E)-2-alkenals, $(E, E)$-alka-2,4-dienals and $n$-alkanals, demonstrating clear oil nature dependencies for the evolution of aldehydes when thermally-stressed according to continuous or discontinuous strategies. 


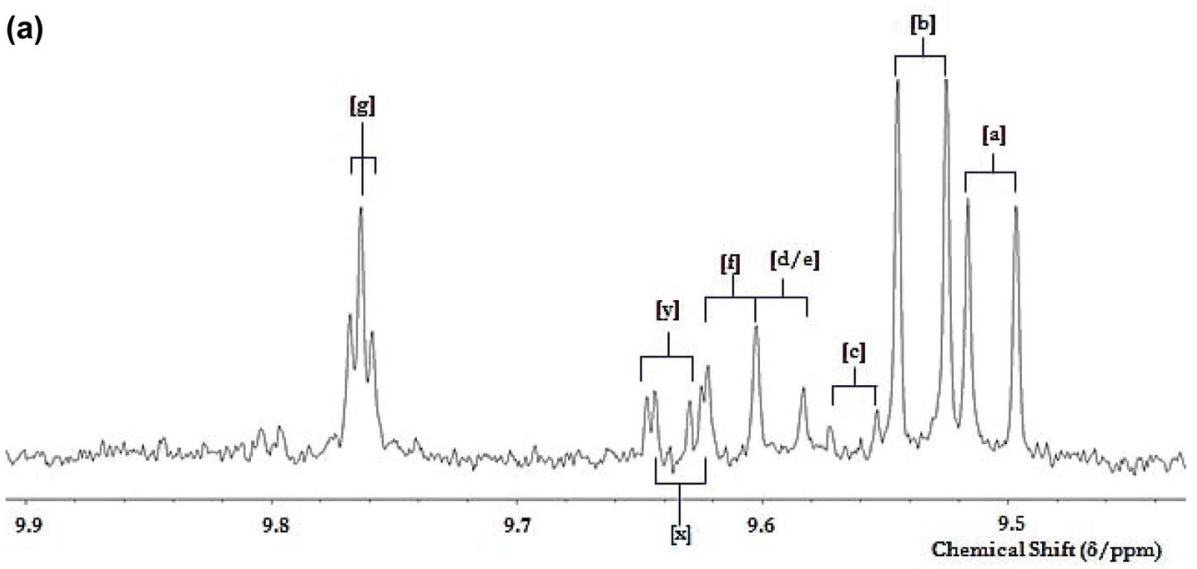

(b)

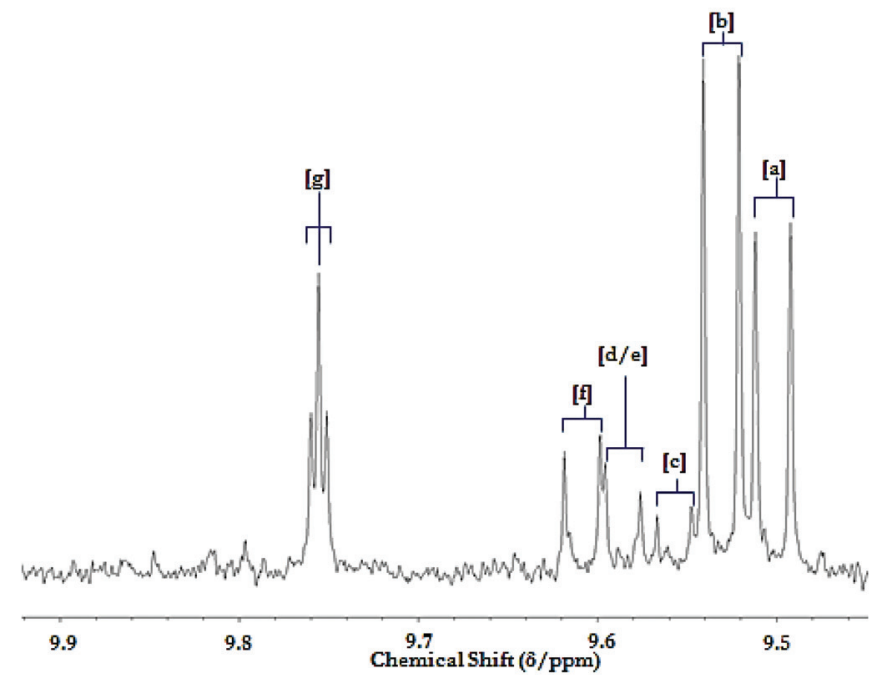

(c)

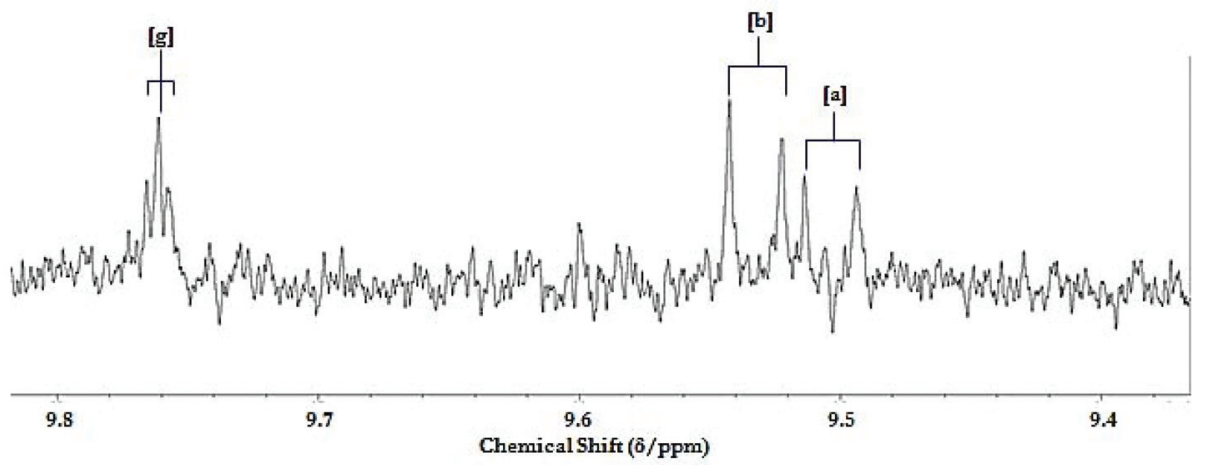

Fig. $12{ }^{1} \mathrm{H}$ NMR analysis of potato chips deep-fried according to repetitive domestic deep repetitive frying sessions. (a) Partial 9.40-9.90 ppm regions of the ${ }^{1} \mathrm{H}$ NMR profile of a $\mathrm{C}^{2} \mathrm{HCl}_{3}$ extract of potato chip samples deep-fried in sunflower oil exposed to $2 \times$ repetitive cycles of 8 sequential $10 \mathrm{~min}$ length deep-frying episodes in a commercially-available domestic deep fryer unit at $170{ }^{\circ} \mathrm{C}$ for two days, followed by 3 further 10 min frying episodes on day 3 (19 in total). Potato chip samples were fried, collected and extracted by the method described in section 2.8. (b) Corresponding partial spectra of the sunflower oil used for these sequential deep-frying sessions; samples were collected for ${ }^{1} \mathrm{H}$ NMR analysis immediately on completion of the above $19^{\text {th }} 10$ min frying session. (c) Corresponding partial spectra of a $\mathrm{C}^{2} \mathrm{HCl}_{3}$ extract of a potato chip sample purchased from a local take-out restaurant. Abbreviations: aldehyde- $\mathrm{CHO}$ function resonance assignments a, b, c, d, e, f and g correspond to those in Fig. 5. Resonances labelled $\mathrm{x}$ and $\mathrm{y}$ tentatively arise from two unassigned classes of $\alpha, \beta$-unsaturated aldehydes. 
To compare variations in LOP composition between our discontinuous and continuous heating protocols, a comparison of the concentrations of all identifiable aldehydic LOPs at a fixed time point (120 min) was undertaken (Fig. 10). Whilst the concentrations of LOPs follow the trend of being proportional to the degree of unsaturation during the continuous heating process, employment of the discontinuous heating strategy exerted a marked impact on their observed concentrations.

ANCOVA analysis of LSM values revealed that the continuous method of thermal stressing only generated significantly greater levels of aldehyde than the discontinuous approach for two of the aldehydes monitored $\left(p<5.59 \times 10^{-3}\right.$ and 0.023 for $(E)$-2-alkenals low-molecular-mass $n$-alkanals respectively) (Table 5). However, it was also clear that any 'betweenmethods' differences manifested were markedly influenced by the nature of the oil product tested. Indeed, all thermal stressing method $\times$ oil nature interaction effects in this model were also very highly significant ( $p<10^{-6}$ for all aldehydes tested), and from Fig. 11, is clear that although sunflower and rapeseed oils generated much lower levels of aldehydic LOPs with the discontinuous heating approach, this method gave rise to higher levels of these toxins in coconut oil; in general, there appeared to be no major differences between these two methods for olive oil.

In the case of both olive and rapeseed oils, the discontinuous heating process produces higher concentrations of lowmolecular-mass $n$-alkanals, 4-oxo- $n$-alkanals, $n$-alkanals and $(E, E)$-2,4-alkadienals at the same time point when compared to those observed during the continuous heating protocol. This suggests a temperature independence of these LOPs being generated (see Fig. S7 and S8 in ESI $\dagger$ ). In practical culinary terms, the heating and cooling of these two oils may result in higher concentrations of these LOPs than by their continuous heating alone. This was to be expected, since during your oil 'rest' periods, the temperature is still quite high, and it takes some time for oil temperature to return to an ambient value. Since MUFA oxidation is a lot slower than that of PUFAs, it also provides more time for this to occur. Interestingly, there was no significant difference between LOPs formed in sunflower oil between discontinuous and continuous heating processes. PUFArich sunflower oil therefore may possess a greater temperatureindependent resistance to oxidation in view of the presence of antioxidants such as alpha-tocopherol (mM levels), which are much more effective against peroxidation at lower temperatures. Coconut oil yielded only very low concentrations of LOPs by NMR analysis when heated according to either process.

Fig. 12(a) shows a typical aldehyde-CHO function regions of the ${ }^{1} \mathrm{H}$ NMR profiles of a $\mathrm{C}^{2} \mathrm{HCl}_{3}$ extract of potato chips deepfried in sunflower when subjected to a repetitive cycles of 19 consecutive 10 min deep-frying episodes within a domestic deep fryer facility at $170{ }^{\circ} \mathrm{C}$ according to section 2.8 . Corresponding partial spectra of the sunflower oil utilised for this purpose is displayed in Fig. 4(b). Aldehydes detectable in the potato chip samples include $(E)$-2-alkenals, $(E, E)$-alka-2,4dienals, 4,5-epoxy-trans-2-alkenals, a combination of 4-hydroxy-/ 4-hydroperoxy-trans-2-alkenals, $(Z, E)$-alka-2,4-dienals and n-alka- nals, along with two unassigned aldehydic proton resonances in the profile shown in (a). Moreover, Fig. 12(c) shows the corresponding ${ }^{1} \mathrm{H}$ NMR spectral region for a sample of fried potato chips obtained from a local Chinese take-out vendor.

\section{Conclusions}

This manuscript compares, for the first time, the effects of continuous and discontinuous heating strategies on the LOP/ FA composition of saturated- and poly/monounsaturated-rich oils using high-resolution (600 MHz) ${ }^{1} \mathrm{H}$ NMR analysis in order to characterise and quantitate the different agents in situ, in a non-destructive fashion. Analysis of differences in acylglycerols indicated that the greatest difference observed between continuous and discontinuous heating processes was for sunflower and rapeseed oils. The susceptibility of the selected oils to thermal oxidation has been evaluated via the characterisation of LOPs from a variety of PUFA, MUFA and SFA sources, with coconut oil exhibiting the most heat resistant activities in this study, with sunflower oil being the most susceptible, as expected. The impact of heating protocol on the identities and quantities of LOPs evolving throughout a $2.0 \mathrm{~h}$ period implies a degree of innate resistance to discontinuous heat-induced autocatalytic oxidation cycles by sunflower oil, when compared to the effects observed with rapeseed and olive oil media. This observation is particularly noteworthy in view of the expectation that this susceptibility would be linked to the degree of unsaturation of the cooking oils investigated.

\section{Conflicts of interest}

The authors declare no conflicts of interest.

\section{Acknowledgements}

The authors are grateful to Doctoral Training Alliance and Kingston University for their support for this project.

\section{References}

1 A. Catalá, Lipid peroxidation of membrane phospholipids generates hydroxy-alkenals and oxidized phospholipids active in physiological and/or pathological conditions, Chem. Phys. Lipids, 2009, 157, 1-11.

2 J. Alberdi-Cedeño, M. L. Ibargoitia, G. Cristillo, P. Sopelana and M. D. Guillén, A new methodology capable of characterizing most volatile and less volatile minor edible oils components in a single chromatographic run without solvents or reagents. Detection of new components, Food Chem., 2017, 221, 1135-1144.

3 M. Grootveld, C. J. L. Silwood, P. Addis and A. Claxson, Health effects of oxidized heated oils, Food Res. Int., 2001, 13, 41-55. 
4 R. P. Venkata and R. Subramanyam, Evaluation of the deleterious health effects of consumption of repeatedly heated vegetable oil, Toxicol. Rep., 2016, 3, 636-643.

5 M. Grootveld, V. R. Rodado and C. J. L. Silwood, Detection, monitoring, and deleterious health effects of lipid oxidation products generated in culinary oils during thermal stressing episodes, InForm, 2014, 25, 614-624.

6 A. Martínez-Yusta, E. Goicoechea and M. D. Guillén, A review of thermo-oxidative degradation of food lipids studied by ${ }^{1} \mathrm{H}$ NMR spectroscopy: influence of degradative conditions and food lipid nature, Compr. Rev. Food Sci. Food Saf., 2014, 13, 838-859.

7 M. D. Guillén and A. Ruiz, Rapid simultaneous determination by proton NMR of unsaturation and composition of acyl groups in vegetable oils, Eur. J. Lipid Sci. Technol., 2003, 105, 688-696.

8 M. D. Guillén and P. S. Uriarte, Simultaneous control of the evolution of the percentage in weight of polar compounds, iodine value, acyl groups proportions and aldehydes concentrations in sunflower oil submitted to frying temperature in an industrial fryer, Food Control, 2012a, 24, 50-56.

9 M. D. Guillén and P. S. Uriarte, Contribution to further understanding of the evolution of sunflower oil submitted to frying temperature in a domestic fryer: study by ${ }^{1} \mathrm{H}$ Nuclear Magnetic Resonance, J. Agric. Food Chem., 2009, 57, 7790-7799.

10 E. Goicoechea and M. D. Guillén, Analysis of hydroperoxides, aldehydes and epoxides by ${ }^{1} \mathrm{H}$ Nuclear Magnetic Resonance in sunflower oil oxidized at 70 and $100{ }^{\circ} \mathrm{C}$, J. Agric. Food Chem., 2010, 58, 6234-6245.

11 M. D. Guillén and E. Goicoechea, Oxidation of corn oil at room temperature: primary and secondary oxidation products and determination of their concentration in the oil liquid matrix from ${ }^{1} \mathrm{H}$ Nuclear Magnetic Resonance data, Food Chem., 2009, 116, 183-192.

12 M. D. Guillén and P. S. Uriarte, Study by ${ }^{1} \mathrm{H}$ NMR spectroscopy of the evolution of extra virgin olive oil composition submitted to frying temperature in an industrial fryer for a prolonged period of time, Food Chem., 2012b, 134, 162-172.

13 B. M. Markaverich, J. R. Crowley, M. A. Alejandro, K. Shoulars, N. Casajuna, S. Mani, A. Reyna and J. Sharp, Leukotoxins diols from ground corncob bedding disrupt estrus cyclicity in rats and stimulate MCF-7 breast cancer cell proliferation, Environ. Health Perspect., 2005, 113, 1698-1704.

14 D. A. Thompson and B. D. Hammock, Dihydroxyoctadecamonoenoate esters inhibit the neutrophil respiratory burst, J. Biosci., 2007, 32, 279-291; B. Chen, A. Han, D. J. McClements and E. A. Decker, Physical structures in soybean oil and their impact on lipid oxidation, J. Agric. Food Chem., 1994, 58, 11993-11999.

15 S. Moumtaz, B. C. Percival, D. Parmar, K. L. Grootveld, P. Jansson and M. Grootveld, Toxic aldehyde generation in and food uptake from culinary oils during frying practices: peroxidative resistance of a monounsaturate-rich algae oil, Sci. Rep., 2019, 9, 1-21.
16 M. J. Picklo, A. Azenkeng and M. R. Hoffmann, Trans-4oxo-2-nonenal potently alters mitochondrial function, Free Radicals Biol. Med., 2011, 50, 400-407.

17 S. P. Hussain, L. S. Hofseth and C. C. Harris, Radical causes of cancer, Nat. Rev. Cancer, 2003, 3, 276-285.

18 R. Guillén-Sans and M. Guzman-Chozas, The thiobarbituric acid (TBA) reaction in foods: a review, Crit. Rev. Food Sci., 1998, 38, 315-330.

19 M. Laguerre, J. Lecomte and P. Villeneuve, Evaluation of the ability of antioxidants to counteract lipid oxidation: existing methods, new trends and challenges, Prog. Lipid Res., 2007, 46, 244-282.

20 A. Le Gresley and J. M. Peron, A semi-automatic approach to the characterisation of dark chocolate by Nuclear Magnetic Resonance and multivariate analysis, Food Chem., 2019, 275, 385-389.

21 E. Falcha, H. W. Anthonsenc, D. E. Axelson and M. Aursanda, Correlation between ${ }^{1} \mathrm{H}$ NMR and traditional methods for determining lipid oxidation of ethyl docosahexaenoate, J. Am. Oil Chem. Soc., 2004, 81, 1105-1110.

22 A. W. D. Claxson, G. E. Hawkes, D. P. Richardson, D. P. Naughton, R. M. Haywood, C. L. Chander, M. Atherton, E. J. Lynch and M. Grootveld, Generation of lipid peroxidation products in culinary oils and fats during episodes of thermal stressing: a high field ${ }^{1} \mathrm{H}$ NMR study, FEBS Lett., 1994, 355, 81-90.

23 A. Martínez-Yusta and M. D. Guillén, Monitoring compositional changes in sunflower oil-derived deep-frying media by ${ }^{1} \mathrm{H}$ Nuclear Magnetic Resonance, Eur. J. Lipid Sci. Technol., 2016, 118, 984-996.

24 B. Nieva-Echevarría, E. Goicoechea and M. D. Guillén, Behaviour of non-oxidized and oxidized flaxseed oils, as models of omega-3 rich lipids, during in vitro digestion. Occurrence of epoxidation reactions, Food Res. Int., 2017, 97, 104-115.

25 B. Nieva-Echevarría, E. Goicoechea, M. J. Manzanos and M. D. Guillén, The influence of frying technique, cooking oil and fish species on the changes occurring in fish lipids and oil during shallow-frying, studied by ${ }^{1} \mathrm{H}$ NMR, Food Res. Int., 2016, 84, 150-159.

26 M. D. Guillén and E. Goicoechea, Detection of primary and secondary oxidation products by Fourier Transform Infrared Spectroscopy (FTIR) and ${ }^{1} \mathrm{H}$ Nuclear Magnetic Resonance (NMR) in sunflower oil during storage, J. Agric. Food Chem., 2007, 55, 10729-10736.

27 M. Grootveld, B. C. Percival and K. L. Grootveld, Chronic noncommunicable disease risks presented by lipid oxidation products in fried foods, Hepatobil. Surg. Nutr., 2018, 7, 305-312.

28 K. M. Schaich, Thinking outside the classical chain reaction box of lipid oxidation, Lipid Technol., 2012, 24, 55-58.

29 R. M. Haywood, A. W. D. Claxson, G. E. Hawkes, D. P. Richardson, D. P. Naughton, G. Coumbarides, J. Hawkes, E. J. Lynch and M. C. Grootveld, Detection of aldehydes and their conjugated hydroperoxydiene precursors in thermally-stressed culinary oils and fats: investigations using high resolution proton NMR spectroscopy, Free Radic. Res., 1995, 22, 441-482. 